\title{
Modeling and Identification of Wear in a Robot Joint under Temperature Uncertainties
}

\author{
André Carvalho Bittencourt, Patrik Axelsson, Ylva Jung, \\ Torgny Brogårdh \\ Division of Automatic Control \\ E-mail: andrecb@isy.liu.se, axelsson@isy.liu.se, \\ ylvju@isy.liu.se, torgny.brogardh@se.abb.com
}

11th October 2010

Report no.: LiTH-ISY-R-2981

Submitted to 8th World Congress of the International Federation of Automatic Control (IFAC 2011)

Milano, Italy, August 28 - September 2, 2011

Address:

Department of Electrical Engineering

Linköpings universitet

SE-581 83 Linköping, Sweden

WWW: http://www.control.isy.liu.se

AUTOMATIC CONTROL

REGLERTEKNIK

LINKÖPINGS UNIVERSITET

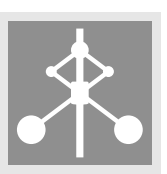

Technical reports from the Automatic Control group in Linköping are available from http://www. control.isy.liu.se/publications. 


\begin{abstract}
This paper considers the problem of wear estimation in a standard industrial robot joint. Motivated by the observation that the wear processes in a robot actuator cause an increase of the friction levels in the joint, static friction observations are used to infer the wear levels. An existing static friction model is extended to include the effects of wear, which are analyzed and modeled. The resulting model can explain friction in a robot joint with respect to changes in speed, load, temperature and wear. Based on this model and a test-cycle that generates static friction observations, a modelbased wear estimator is proposed. The performance of the estimator is found both by means of simulations and experiments on an industrial robot. As it is shown, the method can distinguish the effects of wear even under large temperature variations, opening up for the use of robust joint diagnosis for industrial robots.
\end{abstract}

Keywords: robotics, wear, friction, identification, diagnosis 


\title{
Modeling and Identification of Wear in a Robot Joint under Temperature Uncertainties *
}

\author{
André Carvalho Bittencourt, ${ }^{*}$ Patrik Axelsson, ${ }^{*}$ Ylva Jung* \\ and Torgny Brogårdh ${ }^{* *}$ \\ * Department of Electrical Engineering, Linköpings University, Sweden \\ \{andrecb, axelsson, ylvju\}@isy.liu.se \\ ** ABB Robotics, Västerås, Sweden torgny.brogardh@se.abb.com
}

\begin{abstract}
This paper considers the problem of wear estimation in a standard industrial robot joint. Motivated by the observation that the wear processes in a robot actuator cause an increase of the friction levels in the joint, static friction observations are used to infer the wear levels. An existing static friction model is extended to include the effects of wear, which are analyzed and modeled. The resulting model can explain friction in a robot joint with respect to changes in speed, load, temperature and wear. Based on this model and a test-cycle that generates static friction observations, a model-based wear estimator is proposed. The performance of the estimator is found both by means of simulations and experiments on an industrial robot. As it is shown, the method can distinguish the effects of wear even under large temperature variations, opening up for the use of robust joint diagnosis for industrial robots.
\end{abstract}

Keywords: robotics, wear, friction, identification, diagnosis

\section{INTRODUCTION}

In the manufacturing industry, preventive scheduled maintenance is a common approach used to guarantee the reliability of a robot system, avoiding unpredicted stops. Such scheduling, however, is in general based on the estimated robot's components lifespan and not on its actual conditions. With the development of model-based diagnosis methods, more sophisticated approaches have been proposed for manipulator diagnosis (Caccavale and Villani (2003)). These methods are based on the principle of analytical redundancy, where residuals (deviations between the system outputs and model-based predictions) are monitored to perform fault detection, see Isermann (2005) for an overview on model-based diagnosis methods. A typical approach for residual generation in robotics is the use of nonlinear observers, as presented in McIntyre et al. (2005). Since observers are sensitive to model uncertainties and disturbances, some methods attempt to diminish these effects. In Brambilla et al. (2008) and De Luca and Mattone (2004), nonlinear observers are used together with adaptive schemes while in Caccavale et al. (2009), the authors mix the use of nonlinear observers with support vector machines. The problem has also been approached by the use of neural networks as presented in Vemuri and Polycarpou (2004) and in Eski et al. (2010), where vibration data are used for diagnosis. Parameter estimation is a natural approach because it can use the physical interpretation of the system, see for example Freyermuth (1991).

\footnotetext{
$\star$ This work was supported by $\mathrm{ABB}$ and the Vinnova Industry Excellence Center LINK-SIC at Linköping University.
}

The diagnosis of actuators is a relevant research topic for industrial robots. In the literature, actuator failures are typically considered as abrupt changes in the output torque signals. These fault models can relate to several types of failures such as a motor malfunction, power supply drop or a wire cut. However, such failures are difficult to predict and therefore, even if detected, might still cause damages. An important type of failure is the one related to wear in robot joints. This type of fault develops with time/usage and might be detected at an early stage, allowing for condition-based maintenance.

The wear processes inside a robot joint cause an eventual increase of wear debris in the lubricant. Monitoring the iron content of lubricant samples taken from the robot joint can thus be used as an indication of the joint condition. The study of wear debris particles is known as ferrography and was first introduced by Seifert and Westcott (1972). Since then, the science has evolved and helped to understand wear related phenomena (Roylance (2005)). These techniques are however intrusive and costly, requiring laboratory analyses.

Nevertheless, it is well known that friction changes can follow as a result of wear processes in mechanical systems, see for example Kato (2000). In this paper, it is shown that this is also the case for robot joints. A possible diagnostic solution is therefore to monitor the joint friction. However, due to the complex nature of friction on a robot joint, it is affected also by other factors than wear such as
- temperature,
- velocity,
- force/torque levels,
- joint angle,
- acceleration,
- lubricant/grease properties. 
When designing a diagnostic method, it is necessary to understand the effects of the different variables to be able to distinguish them from wear effects. In Bittencourt et al. (2010) a static friction ${ }^{1}$ model is presented that can represent the effects of speed, temperature and load. Based on empirical observations, this model is in this paper extended to include wear effects. With the proposed model, it is possible to predict the behavior of the observed joint friction over wide operating conditions, opening up for robust diagnosis.

The static friction can not, however, be directly measured in a robot joint. As presented in this paper though, friction levels can be estimated at a given speed using dedicated experiments. Friction can therefore be monitored at a set of chosen speeds using a pre-defined test cycle. When designing test cycles, it is important to distinguish between cycles used for the development of diagnosis methods and cycles used for diagnosis of a robot in an industrial installation. In the later case, the cycle must take only a short time to perform. This means that the cycle must be custom designed for the estimation of the desired quantity. In the following, it is shown that it is possible to obtain an efficient test cycle if correct speed levels are used, even under large temperature variations.

The main contribution of this paper is a proposed model-based wear estimation method and analysis of the best speed region for wear estimation under joint temperature uncertainties. The method is based on static friction observations achieved from a test cycle, an empirical wear model and the friction model presented in Bittencourt et al. (2010). The estimator is defined in a prediction error sense, minimizing the difference between the observed friction, $\tau_{f}$, and the model-based prediction, that is

$$
\hat{\mathrm{w}}=\arg \min _{\mathrm{w}} V\left(\tau_{f}-\hat{\tau}_{f}\left(\dot{\varphi}, \tau_{m}, T, \mathrm{w}\right)\right),
$$

where $\hat{\tau}_{f}(\cdot)$ are friction predictions given by a model, $V(\cdot)$ is a cost function, $\varphi$ is the vector of joint angles, $\dot{\varphi}=\frac{\mathrm{d}}{\mathrm{d} t} \varphi$, $\tau_{m}$ is the manipulated load torque and $T$ is the joint temperature, see also Fig. 1(b). The study is based on observed static friction in joint 2 of an ABB IRB 6620 industrial robot, see Fig. 1(a). Since joint temperature is seldom measured in industrial applications, its effects are considered assuming $T$ as a random variable with an unknown distribution function but known lower and upper limits.

The paper is organized as follows. Section 2 presents a method to observe static friction in a robot joint that can be used to define a test-cycle. Section 3 discusses the static friction model presented in Bittencourt et al. (2010). In Section 4, this model is extended to include the effects of wear, which are analyzed and modeled based on empirical observations. Section 5 presents the proposed wear estimation method and evaluates its accuracy over the robot joint speed range. Section 6 presents a case study based on real data. The conclusions and proposals for further research are presented in Section 7.

\footnotetext{
1 The term static friction refers to observed friction at constant speeds, without transient movements.
}

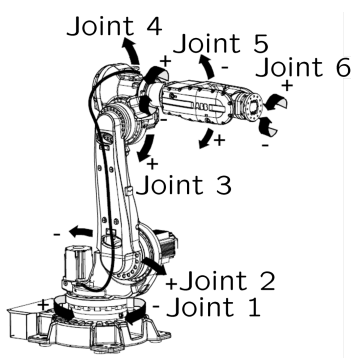

(a) ABB IRB 6620 robot with $150 \mathrm{~kg}$ payload and $2.2 \mathrm{~m}$ reach.

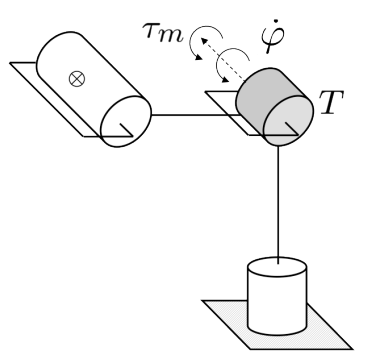

(b) Schematics of the 3 first joints including the variable definitions for joint 2 .
Fig. 1. The study is based on joint 2 of the ABB robot IRB 6620. $\dot{\varphi}$ is the joint angular speed, $T$ the joint temperature, the manipulated load torque $\tau_{m}$ is the load torque component aligned to the joint degree of freedom.

\section{STATIC FRICTION OBSERVATIONS THROUGH EXPERIMENTS}

A manipulator is a multivariable, nonlinear system that can be described in a general manner through the rigid multi body dynamic model

$$
M(\varphi) \ddot{\varphi}+C(\varphi, \dot{\varphi})+\tau_{g}(\varphi)+\tau_{f}(\dot{\varphi})=u
$$

where $\ddot{\varphi}=\frac{\mathrm{d}}{\mathrm{d} t} \dot{\varphi}, M(\varphi)$ is the inertia matrix, $C(\varphi, \dot{\varphi})$ relates to speed dependent terms (e.g. Coriolis and centrifugal), $\tau_{g}(\varphi)$ are the gravity-induced joint torques and $\tau_{f}$ contains the joint friction components. The system is controlled by the input torque, $u$, applied by the joint motor (in the experiments the torque reference from the servo was measured $^{2}$ ).

For single joint movements $(C(\varphi, \dot{\varphi})=0)$ under constant speed $(\ddot{\varphi} \approx 0)$, Eq. (2) simplifies to

$$
\tau_{g}(\varphi)+\tau_{f}=u \text {. }
$$

The resulting applied torque $u$ drives only friction and gravity-induced torques. The required torques to drive a joint in forward, $u^{+}$, and reverse, $u^{-}$, directions at the constant speed level $\overline{\dot{\varphi}}$ and at a joint angle value $\bar{\varphi}$ (so that $\tau_{g}(\bar{\varphi})$ is equal in both directions), are

$$
\begin{aligned}
\tau_{f}(\overline{\dot{\varphi}})+\tau_{g}(\bar{\varphi}) & =u^{+} \\
\tau_{f}(-\overline{\dot{\varphi}})+\tau_{g}(\bar{\varphi}) & =u^{-} .
\end{aligned}
$$

In the case an estimate of $\tau_{g}(\bar{\varphi})$ is available, it is possible to isolate the friction component in each directions using Eq. (4). If such estimate is not possible (e.g. not all masses are completely known), $\tau_{f}$ can still be achieved in the case that $\tau_{f}$ is independent of the rotation direction. Subtracting the equations yields

$$
\tau_{f}(\overline{\dot{\varphi}})-\tau_{f}(-\overline{\dot{\varphi}})=u^{+}-u^{-}
$$

and if $\tau_{f}(-\overline{\dot{\varphi}})=-\tau_{f}(\overline{\dot{\varphi}})$, the resulting direction independent friction is:

$$
\tau_{f}(\bar{\varphi})=\frac{u^{+}-u^{-}}{2}
$$

In the experiments, each joint is moved with the desired speed in both directions around a given joint angle $\bar{\varphi}$. Fig. 2 shows the measured joint angle-, speed- and torque ${ }^{3}$

\footnotetext{
2 It is known that using the torque reference from the servo as a measure of the joint torque might not always hold because of the temperature dependence of the torque constant of the motors. The
} 


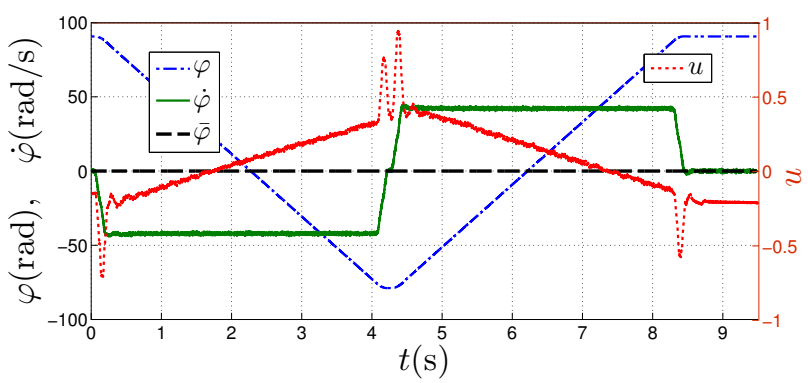

Fig. 2. Excitation signals used for the static friction estimation at $\dot{\varphi}=42 \mathrm{rad} / \mathrm{s}$.

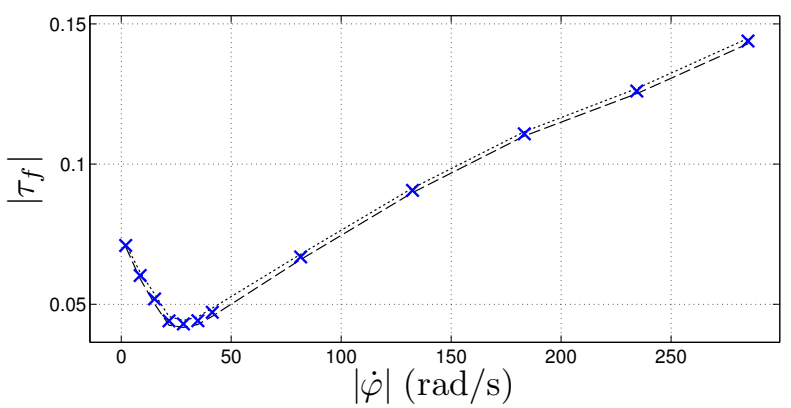

Fig. 3. Static friction curve. Crosses indicate friction levels achieved using Eq. (5), with the assumption that friction is direction independent. Dotted/dashed lines indicate friction levels achieved using Eq. (4a) and Eq. (4b) respectively.

signals sampled at $2 \mathrm{kHz}^{4}$ for $\overline{\dot{\varphi}}=42 \mathrm{rad} / \mathrm{s}$ around $\bar{\varphi}=0$. The constant speed data is segmented around $\bar{\varphi}$ and the static friction levels can be achieved using Eq. (4) or (5). With the experimental setup used, the time needed for measurements and computations of the friction level for one joint at one speed was in average $14 \mathrm{~s}$.

The procedure can be repeated for several different speed levels and can be used to generate test cycles for friction monitoring, where the choice of speed set is a design criteria. The friction values achieved over the whole joint speed range can then be presented in a static friction curve, sometimes referred to as a Stribeck curve, see Fig. 3.

As seen in Fig. 3, there is only a small direction dependency of friction for the investigated joint. Therefore, in this paper, friction levels are achieved using Eq. (5), which is not influenced by deviations in the gravity model of the robot.

Remark 1. Throughout the paper, friction values obtained using the method presented in this section are named friction observations.

\section{STATIC FRICTION MODEL}

In a robot joint, the complex interaction of components such as gears, bearings, sealings and shafts which are rotating/sliding at different velocities, makes physical modeling

deviations are however considered to be small and are neglected during the experiments.

3 Throughout the paper all torques are normalized to the maximum manipulation torque at low speed.

4 Similar results have been experienced with sampling rates down to $220 \mathrm{~Hz}$.

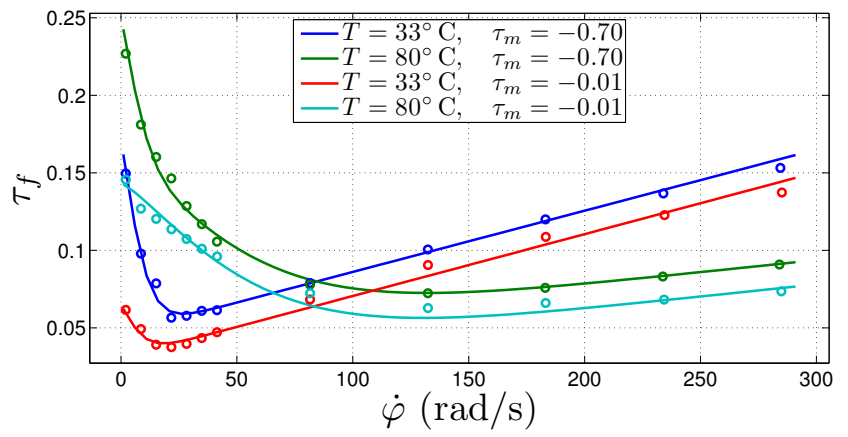

Fig. 4. Observed static friction curves (circles) and model-based predictions (lines) for low and high values of $T$ and $\tau_{m}$.

of friction difficult (see Al-Bender and Swevers (2008) for a comprehensive discussion on friction physics and first principle friction modeling). However, it has been shown that empirically designed fricition models can be used also for complex robot joints. In Bittencourt et al. (2010), a static friction model that can cope with temperature and joint load torque levels is presented. As shown, this model can be used to predict the normal behavior of static friction under broad operation conditions. The model is developed from the so called LuGre model (Åström and Canudas-de Wit (2008)) and empirical observations, taking the form:

$$
\begin{aligned}
& \tau_{f}\left(\dot{\varphi}, \tau_{m}, T\right)= \\
& \left\{F_{c, 0}+F_{c, \tau_{m}}\left|\tau_{m}\right|\right\}+F_{s, \tau_{m}}\left|\tau_{m}\right| e^{-\left|\frac{\dot{\varphi}}{\dot{\varphi}_{s, \tau_{m}}}\right|^{1.3}}+ \\
& +\left\{F_{s, 0}+F_{s, T} T\right\} e^{-\left|\frac{\dot{\varphi}}{\left\{\dot{\varphi}_{s, 0}+\dot{\varphi}_{s, T} T\right\}}\right|^{1.3}}+ \\
& +\left\{F_{v, 0}+F_{v, T} e^{\frac{-T}{T_{\mathrm{Vo}}}}\right\} \dot{\varphi},
\end{aligned}
$$

where $\tau_{m}$ is the load torque component manipulated by the joint and $T$ is the joint temperature, see Fig. 1(b). The remaining parameters are used to model the friction behavior. The terms in (6a) describe the effects of $\tau_{m}$, which are more significant at low speeds; the terms in (6b) and (6c) describe the effects of $T$ at low and high speed ranges respectively (see Bittencourt et al. (2010) for a more detailed discussion). The identified friction model parameters values used in this paper are shown in Table 1.

To illustrate the model behavior, Fig. 4 presents observed and model-based predictions of friction curves for high and low values of $\tau_{m}$ and $T$. Notice the effects of $\tau_{m}$, which give an offset increase of the whole curve together with an exponential-like increase at speeds below $25 \mathrm{rad} / \mathrm{s}$. The effects of $T$ can be seen as an exponential increase at speeds below $80 \mathrm{rad} / \mathrm{s}$ and a decrease of the curve slope at higher speeds. Notice further that for such temperature changes there is a speed range where the effects are less pronounced, in this case around $80 \mathrm{rad} / \mathrm{s}$.

\section{WEAR - ANALYSIS AND MODELING}

It might be difficult to fully comprehend the effects of wear in a robot joint. Monitoring the system until a failure takes place is a costly and time consuming task. Furthermore, resolving for coupled effects between wear, temperature, load and other parameters would require even more longterm experiments. With the objective of understanding these effects, accelerated wear tests were performed with a robot joint while friction curves were observed periodically. 
Table 1. Identified parameters for the model (6).

\begin{tabular}{c|c|c|c|c|c|c|c|c|c|c}
\hline$F_{c, 0}$ & $F_{c, \tau_{m}}$ & $F_{s, 0}$ & $F_{s, \tau_{m}}$ & $F_{s, T}$ & $F_{v, 0}$ & $F_{v, T}$ & $\dot{\varphi}_{s, 0}$ & $\dot{\varphi}_{s, \tau_{m}}$ & $\dot{\varphi}_{s, T}$ & $T_{\mathrm{Vo}}$ \\
\hline $3.0410^{-2}$ & $2.3210^{-2}$ & $-2.4410^{-2}$ & $1.2810^{-1}$ & $1.6910^{-3}$ & $1.2910^{-4}$ & $1.3110^{-3}$ & -25.00 & 9.07 & 1.00 & 21.00 \\
\hline
\end{tabular}

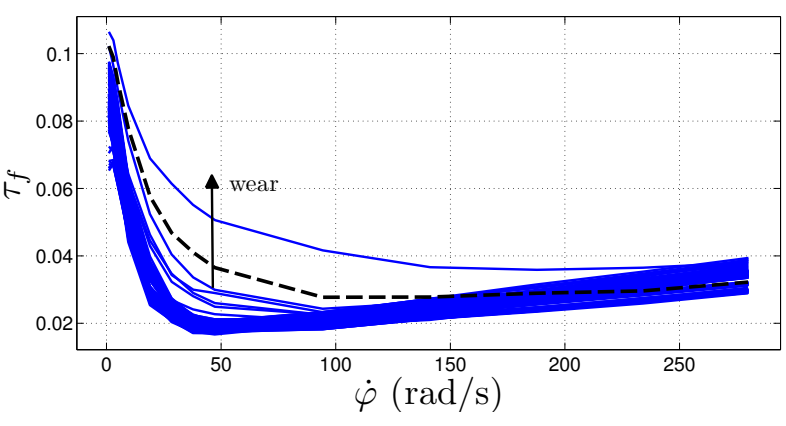

Fig. 5. Effects of wear in friction curves observed at regular intervals under accelerated wear tests taken under constant load- and temperature conditions. The dashed line relates to the wear level at which an alarm should be generated.

The results of such an experiment is shown in Fig. 5, with observed friction curves obtained at the same loadand temperature levels. As can be noticed, the effects of wear appear first in the low speed region, in this case, up to about $150 \mathrm{rad} / \mathrm{s}$. In Fig. 5, the dashed line indicates the samples associated with a wear level that gear experts find relevant for issuing an alarm. Up to this point the changes appear mostly in the low speed range. If the accelerated wear tests proceed, the friction curve is also affected at higher speed levels. A direct comparison with Fig. 4 reveals that the friction changes caused by increased wear are in the same magnitude as the changes caused by load/temperature but with different speed dependence. Therefore, it might be possible to obtain a selective identification of wear in a robot joint.

\subsection{Wear Modeling}

The effects of wear in Fig. 5 can be isolated assuming that the effects of load/temperature are independent of those caused by wear. A wear profile $\tilde{\tau}_{f}$ is defined by subtracting a friction curve observed before the accelerated wear tests started from the ones obtained from the same robot with accelerated tests. The resulting wear profile from the accelerated wear test in Fig. 5 can be seen in Fig. 6, where the curves are presented along a time index $k$, indicating the length of the accelerated tests. In the figure, the dashed line at time-index $k=80$ indicates the samples when it is time for a diagnostic system to generate an alarm.

As can be noticed, the effects of wear appear as an increase of the exponential-like behavior of the friction curves up $100 \mathrm{rad} / \mathrm{s}$ and decrease of the velocity slope dependency at higher speeds. Introducing $\mathrm{w}$ as a wear parameter, the observations support the choice of a model structure for the wear profile as

$$
\tilde{\tau}_{f}(\mathrm{w})=F_{s, \mathrm{w}} \mathrm{w} e^{-\left|\frac{\dot{\varphi}}{\dot{\varphi} s, \mathrm{ww}}\right|^{1.3}}+F_{v, \mathrm{w}} \mathrm{w} \dot{\varphi} .
$$

The model represents wear effects with an exponentialand a velocity dependent terms, with 3 parameters. The model parameters cannot be directly identified since the wear quantity $w$ is not measurable. To overcome this, $w$ is defined with values between $[0,100]$, relative to a failure

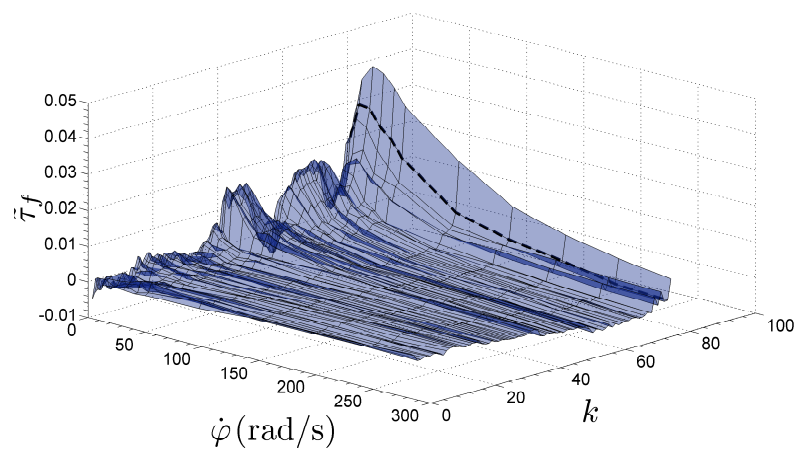

Fig. 6. Friction wear profile $\tilde{\tau}_{f}$ computed from the data in Fig. 5. The dashed line indicates the experiment where an alarm should be given.

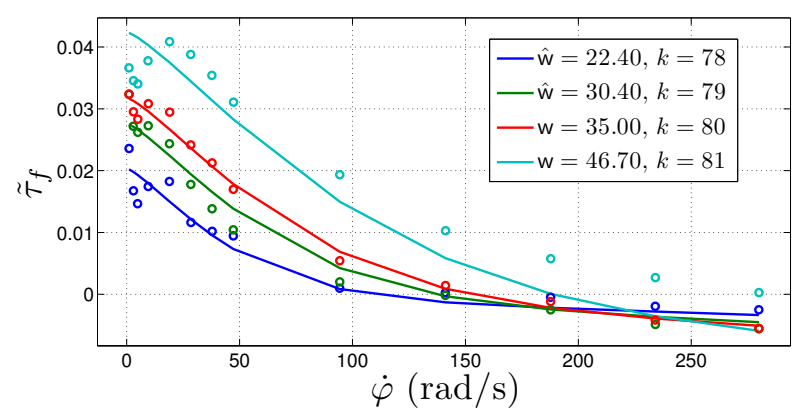

Fig. 7. Measured wear profile (circles) and model-based predictions (lines).

state, the value $\mathbf{w}=35$ is chosen as a reference for the wear effects associated with an alarm, at $k=80$ in Fig. 6 . With this convention, the parameters for (7) are identified using the wear profile data $\tilde{\tau}_{f}$ for the curve at $k=80$. The values achieved are

$$
F_{s, \mathrm{w}}=9.1010^{-3}, \quad F_{v, \mathrm{w}}=-5.310^{-7}, \quad \dot{\varphi}_{s, \mathrm{w}}=2.20 .
$$

With the chosen parameters, an estimate $\hat{w}$ is obtained using the data for the whole wear profile in Fig. 6. Using the identified wear values, the wear profile given by model predictions from (7) and observations are presented for the interval $k=[78,81]$ in Fig. 7 . As can be noticed, the model can fairly well predict the behavior of $\tilde{\tau}_{f}$.

Under the assumption that the effects of load/temperature are independent of those caused by wear, it is possible to extend the model given in (6) to include the effects of wear as

$$
\tau_{f}\left(\dot{\varphi}, \tau_{m}, T, \mathrm{w}\right)=\tau_{f}\left(\dot{\varphi}, \tau_{m}, T\right)+\tilde{\tau}_{f}(\mathrm{w}),
$$

where $\tau_{f}\left(\dot{\varphi}, \tau_{m}, T\right)$ is given by $(6)$ and $\tilde{\tau}_{f}(\mathrm{w})$ is described in (7). With parameters given by Table 1 and Eq. (8), Fig. 8 presents the friction predictions given by the proposed model at $T=40^{\circ} \mathrm{C}$ and $\tau_{m}=0.10$ for wear values in the range $\mathbf{w}=[0,40]$. Notice that the effects are concentrated to the speed range of $[0,150] \mathrm{rad} / \mathrm{s}$. As previously, the dashed line in Fig. 8 indicates an alarm level for the wear, it has a friction increase of 0.017 at $47 \mathrm{rad} / \mathrm{s}$ and $\mathrm{w}=35$. 


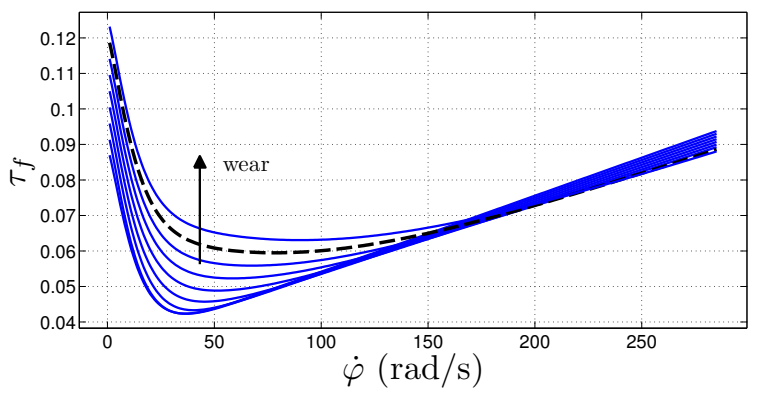

Fig. 8. Increase of wear levels given by the model (9). The dashed line relates to the wear level at which an alarm should be generated.

\section{A MODEL-BASED WEAR ESTIMATOR}

Considering that $\dot{\varphi}, \tau_{m}$ and $T$ can be measured/estimated, it is possible to define a wear estimator in a prediction error sense as in Eq. (1)

$$
\hat{\mathrm{w}}=\arg \min _{\mathrm{w}} V\left(\tau_{f}-\hat{\tau}_{f}\left(\dot{\varphi}, \tau_{m}, T, \mathrm{w}\right)\right),
$$

where $\tau_{f}$ is observed through the procedure presented in Section 2 and the predictions $\hat{\tau}_{f}$ are given by the model in (9). Since the model is not invertible with respect to w, the problem should be solved by nonlinear identification methods.

In industrial applications, joint temperature measurements are seldom available. In order to solve this problem it is proposed to consider joint temperature to be a random variable, with a certain probability distribution function $p(T)$. The distribution function $p(T)$ is assumed to be unknown, but with known lower and upper limits $\underline{T}, \bar{T}$. For a robot operating in a controlled indoor environment, $\underline{T}$ would be minimum room temperature while $\bar{T}$ is given by the maximum room temperature and self heating of the joint because of actuator losses.

To include the effects of $T$ in the estimator, Eq. (1) is solved for $N$ realizations of the considered random, $T$. The estimator assumes that $p(T)$ is constant in the given temperature range and samples are drawn with equal probabilities over $[\underline{T}, \bar{T}]$. The expected value of the resulting $N$ estimates is then taken as the wear estimate,

$$
\begin{aligned}
\hat{\mathrm{w}}_{i} & =\arg \min _{\mathrm{w}} V\left(\tau_{f}-\hat{\tau}_{f}\left(\dot{\varphi}, \tau_{m}, T_{i}, \mathrm{w}\right)\right) \\
\hat{\mathrm{w}} & =E\left[\left\{\hat{\mathrm{w}}_{N}\right\}\right], \quad T_{i} \sim \mathcal{U}(\underline{T}, \bar{T}), \quad i=1, \ldots, N
\end{aligned}
$$

here $\hat{\tau}_{f}\left(\dot{\varphi}, \tau_{m}, T_{i}, \mathrm{w}\right)$ is given by $(9)$ and $\left\{\hat{\mathrm{w}}_{N}\right\}$ denotes the sequence $\left\{\hat{\mathrm{w}}_{1}, \ldots, \hat{\mathrm{w}}_{N}\right\}$.

To solve the minimization problem in (10a), grid search was used with a square error cost function $V(\cdot)$ for the nonlinear static greybox friction model in (9) with parameters given in Table 1 and in (8). To evaluate (10b), a Monte Carlo simulation was carried out. For a given observation of $\tau_{f}$, it takes $N$ samples $T_{i}$ from $\mathcal{U}(\underline{T}, \bar{T})$, yielding $N$ estimates $\left\{\hat{w}_{N}\right\}$. The expected value of the sequence $\left\{\hat{\mathrm{w}}_{N}\right\}$ is then finally taken as the wear estimate $\hat{w}$.

\subsection{Statistical Properties}

To evaluate the behavior of the proposed estimator, the static friction observations are assumed to follow the model

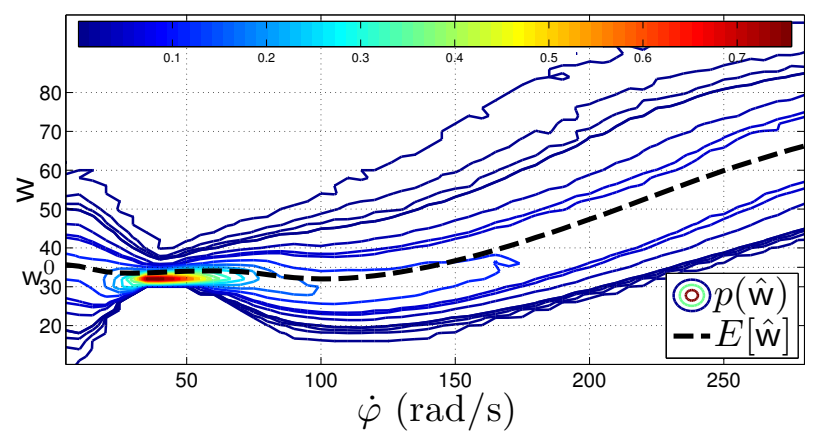

Fig. 9. Contour plot of the estimated probability distribution function of $\hat{w}$ as a function of speed. Notice the sharper densities in the interval $[35,55] \mathrm{rad} / \mathrm{s}$.

$$
\begin{array}{r}
\tau_{f}=\tau_{f}\left(\dot{\varphi}, \tau_{m}, T, \mathrm{w}^{0}\right)+e, \\
e \sim \mathcal{N}\left(0, \sigma_{e}\right), \quad T \sim \mathcal{N}\left(\mu_{T}, \sigma_{T}\right)
\end{array}
$$

where $\tau_{f}(\cdot)$ is generated according to the model (9) with parameters given in Table 1 and in (8), and where $e$ is additive measurement noise. The stochastic properties of $e$ are motivated by experimental studies of the estimation method presented in Section 2. For the considered joint, the estimated standard deviation is $\sigma_{e}=0.0015$. The wear level is defined as $\mathrm{w}^{0}=35$, which means $35 \%$ wear in relation to a failure state, and is related to the alarm level for the wear as discussed in Section 4. Temperature is considered to follow the normal distribution $T \sim \mathcal{N}\left(\mu_{T}, \sigma_{T}\right)$, with mean and standard deviation $\left[\mu_{T}, \sigma_{T}\right]=[40,3]^{\circ} \mathrm{C}$. The chosen distribution illustrates the scenario where friction observations are always taken after the same sequence of events during the day, for instance between two production shifts, in a room with controlled environment temperature. The large standard deviation is used to cope with, amongst others, variations of room temperature and variations of self-heating caused by the different loses for the operations of the robot. The observations of friction torques $\tau_{f}$ obtained from the model in (11) are used as input to the estimator defined in (10), with $N=200$ and $[\underline{T}, \bar{T}]=[30,50]^{\circ} \mathrm{C}$.

To evaluate the performance of the estimation method, 10,000 simulations were made at 56 different speeds in the range $\dot{\varphi}=[0,280] \mathrm{rad} / \mathrm{s}$. The estimation is performed separately for each speed point to assess the method performance along the achievable speed range. Fig. 9 presents the estimated probability distribution function of the estimates $\hat{w}$ with respect to speed in a contour plot, together with their expected values. As it can be noticed, the variability of the estimates is smaller in the speed range $[35,55] \mathrm{rad} / \mathrm{s}$. Fig. 10 presents the estimated bias over speed. Notice the small bias at speeds up to $150 \mathrm{rad} / \mathrm{s}$ and the monotonic increase at higher speeds. The results are directly related to the behavior of the modeled wear and temperature effects for the considered $p(T)$ (recall Fig. 4 and Fig. 8).

Variance. From the simulation studies, it is possible to estimate the resulting variance of the estimator. To assess its performance, the Cramer-Rao bound (CRB) is also computed according to Van Trees (2001).

For the observation model defined in (11) introduce 


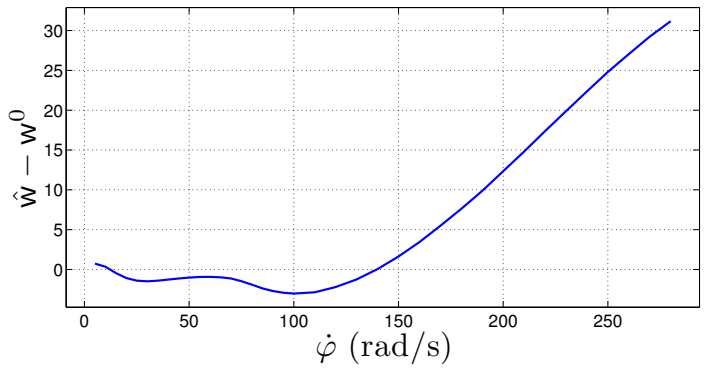

Fig. 10. Estimated bias of $\hat{w}$ along the robot speed range. Notice the better performance in the interval $[50,90] \mathrm{rad} / \mathrm{s}$.

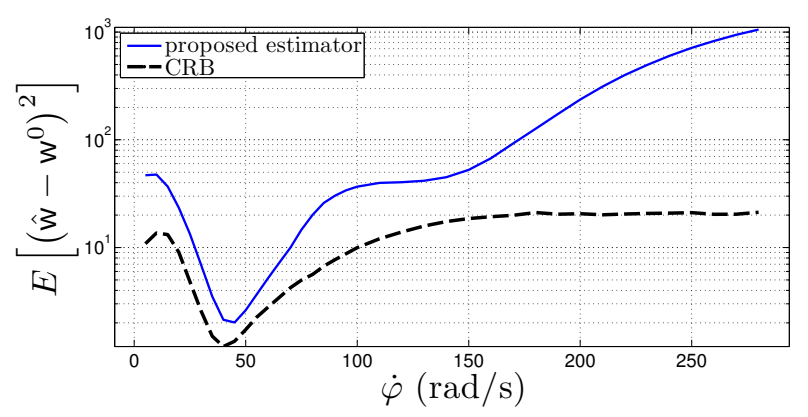

Fig. 11. Variance of the estimator, shown together with its CRB in semi-log scale. Notice the considerable better performance at velocities in the interval $[35,55] \mathrm{rad} / \mathrm{s}$.

$$
\bar{p}\left(\tau_{f} \mid \mathrm{w}\right) \triangleq \iint p\left(\tau_{f} \mid \mathrm{w}, e, T\right) p(e) p(T) \mathrm{d} e \mathrm{~d} T,
$$

where $p(\cdot)$ denotes a probability distribution function. Eq. (12) is the marginalization of the effects of measurement noise and random temperature (Van Trees (2001)). Using $\bar{p}\left(\tau_{f} \mid \mathrm{w}\right)$, the CRB for any unbiased estimator can be defined as

$$
\begin{aligned}
& E\left[\left(\hat{\mathrm{w}}-\mathrm{w}^{0}\right)^{2}\right] \geq M^{-1} \\
& M=E\left[\left(\frac{\partial}{\partial \mathrm{w}} \log \bar{p}\left(\tau_{f} \mid \mathrm{w}\right)\right)^{2}\right]
\end{aligned}
$$

Since there is no analytical solution for (12), Monte Carlo Integration (MCI) is used to compute it numerically. The derivative in $(13 \mathrm{~b})$ is approximated numerically with the central difference,

$$
\frac{\log \bar{p}\left(\tau_{f} \mid \mathrm{w}^{0}+h\right)-\log \bar{p}\left(\tau_{f} \mid \mathrm{w}^{0}-h\right)}{2 h}
$$

for $h=0.1$.

The CRB is shown together with the variance for the proposed estimator in Fig. 11. As can be noticed, the variance is high at low and high speeds. The estimator can however approximate the CRB at the speed region $[35,55] \mathrm{rad} / \mathrm{s}$. This illustrates the relevance of a correct choice of speed points to observe friction for wear identification.

\section{CASE STUDY}

Gathering enough informative data related to wear from the field is inviable since robots will rarely have wear related problems. The alternative of running accelerated wear tests is also difficult since it might take several months before any wear effects can be seen and it is difficult to obtain reliable statistics without extremely

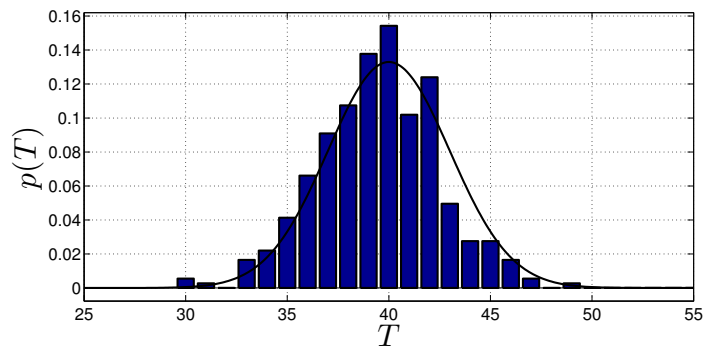

Fig. 12. Reference (line) and achieved (histogram) temperature distributions for the data used in the case study.

high cost of running several robots. Moreover, temperature studies are also challenging since the thermal time constant of a large robot is several hours. An alternative is proposed that considers two data sets:

Wear profile The first data set is taken from a robot that undergoes accelerated wear tests but with constant loadand temperature levels. Under the assumption that wear is not load/temperature dependent, a wear profile $\tilde{\tau}_{f}(k)$ is defined from these data as in Subsection 4.1, where $k$ is a time index. More specifically, the data in Fig. 6 is used.

Temperature effects The second data set is related to friction curves observed on another robot of the same type with no wear effects but at several different temperature conditions. Joint temperature measurements were registered during the experiments and each friction curve in the set is associated with a joint temperature $T$. The notation $\tau_{f}^{0}(T)$ is then used to denote the friction curve in this data set related to the joint temperature $T$.

Under the assumption that wear effects are independent of those caused by temperature, the static friction observation model is defined as

$$
\tau_{f}^{*}(k)=\tilde{\tau}_{f}(k)+\tau_{f}^{0}(T) .
$$

Using this framework, it is possible to generate faulty friction observations under different temperature conditions. Notice that these data are not analytically generated, but actually based on static friction observations.

To achieve a desired temperature distribution for the observations $\tau_{f}^{*}(k)$, the friction data $\tau_{f}^{0}(T)$ are chosen according to the associated measured joint temperature $T$. The reference distribution considered for this case study is the same used in Section 5.1, $\mathcal{N}\left(\mu_{T}, \sigma_{T}\right)$, with mean and standard deviation $\left[\mu_{T}, \sigma_{T}\right]=[40,3]^{\circ} \mathrm{C}$. The reference and achieved temperature distributions for the data used in this case study can be seen in Fig. 12 .

Wear is estimated with the generated data using the method given in (10), with $[\underline{T}, \bar{T}]=[30,50]^{\circ} \mathrm{C}$ and $N=200$. The estimates are generated separately for each speed to assess its performance for the different speeds. The result is presented in Fig. 13, showing the wear estimate curves as function of the speed and $k$.

In accordance to the simulation studies presented in Section 5.1, the estimates have larger variance at low and high speeds with increasing bias at high speeds. The estimation performance was found to be good at speeds close to $\dot{\varphi}=40 \mathrm{rad} / \mathrm{s}$. In this speed region, $\hat{w}$ increases according to 


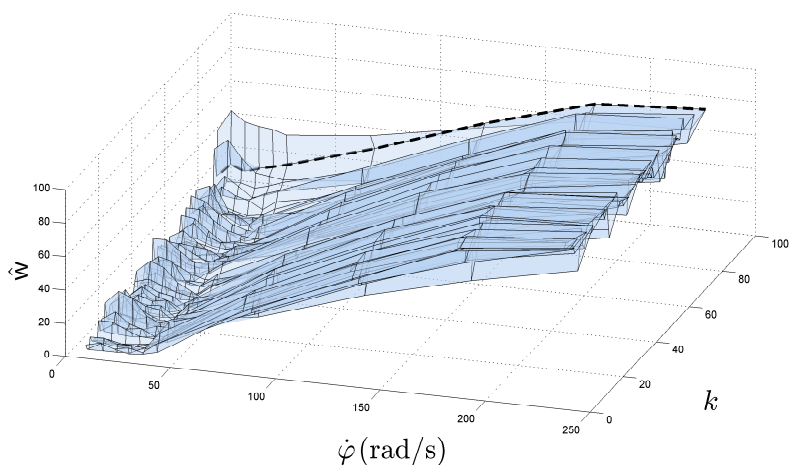

Fig. 13. Estimated wear levels for the different speeds and timeindices. The dashed line indicates the sample where an alarm should be raised.

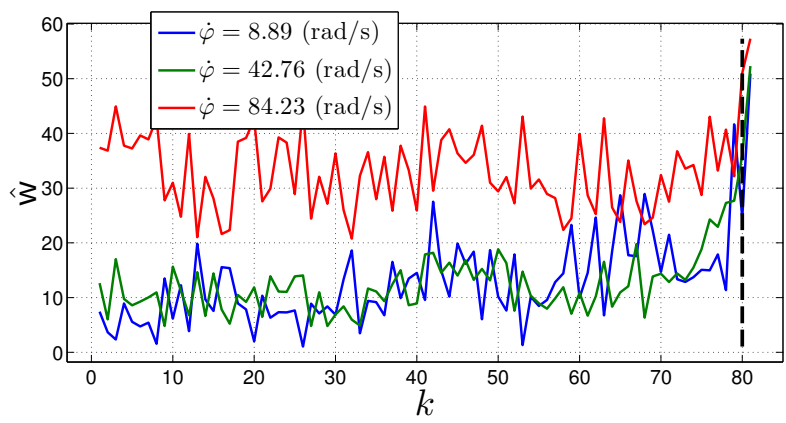

Fig. 14. Estimated wear levels as function of $k$ at selected speed points. The dashed line indicates the friction increase that should raise an alarm.

the wear level behavior but with slight biases. To illustrate this behavior further, Fig. 14 presents the wear estimates for a set of speeds, showing that the best performance is obtained for $\dot{\varphi}=42.76 \mathrm{rad} / \mathrm{s}$. At this speed, the estimate at the sample in which an alarm should be raised, $k=80$, is $\hat{w}=35.99$, in accordance to Section 4 . At this velocity region, despite the bias in the beginning, even a simple threshold set at $w=35$ could be used to detect the critical wear increase.

\section{CONCLUSIONS}

The paper has presented a method to estimate wear in a robot joint. The method is based on the fact that the wear processes inside a robot joint causes increases of the joint friction. To explore this, the effects of wear on the behavior of static friction have been analyzed and modeled. A model-based wear estimator was defined based on static friction observations from a test-cycle and an extended friction model that can represent friction with respect to speed, load, temperature and wear. Since joint temperature is seldom measured in industrial applications, its effects were considered in the estimator by treating it as a random variable with unknown distribution and know lower and upper limits.

A test-cycle was proposed that can be used to achieve static friction observations at a pre-defined set of speeds. In order to find the optimal speed region to perform wear estimation, simulations were used to asses the variance of the estimates in presence of large temperature variations. The simulation results were supported by a case study based on real data. As it was shown, the wear estimates in the optimal speed region could easily be used to perform fault detection, allowing for robust condition based maintenance of industrial robots. This optimal speed region is however narrow, emphasizing the relevance of a correct choice of speed values. If the friction observations are made on a robot installed in a manufacturing line, there is the tradeoff between making the test cycle both accurate and short. In case more time can be spent for the diagnosis routine, the accuracy of the estimation could be improved by observing friction at various speeds around the optimal one.

The work will proceed with studies of the method when also variations of the torque levels and the lubricant properties take place. Later studies will moreover be made of the accuracy of the developed method for different types of robot joints. Especially interesting is to find out the friction- and wear behavior of different gear types. Investigations will also be made to see if it is possible to perform reliable wear estimations without using custom designed experiments.

\section{REFERENCES}

Al-Bender, F. and Swevers, J. (2008). Characterization of friction force dynamics. IEEE Control Systems Magazine, 28(6), 64-81.

Åström, K.J. and Canudas-de Wit, C. (2008). Revisiting the lugre friction model. Control Systems Magazine, IEEE, 28(6), 101-114.

Bittencourt, A.C., Wernholt, E., Sander-Tavallaey, S., and Brogårdh, T. (2010). An extended friction model to capture load and temperature effects in robot joints. In The 2010 IEEE/RSJ International Conference on Intelligent Robots and Systems.

Brambilla, D., Capisani, L., Ferrara, A., and Pisu, P. (2008). Fault detection for robot manipulators via second-order sliding modes. Industrial Electronics, IEEE Transactions on, 55(11), 3954-3963.

Caccavale, F., Cilibrizzi, P., Pierri, F., and Villani, L. (2009). Actuators fault diagnosis for robot manipulators with uncertain model. Control Engineering Practice, 17(1), 146 - 157.

Caccavale, F. and Villani, L. (2003). Fault diagnosis for industrial robots. In Fault Diagnosis and Fault Tolerance for Mechatronic Systems:Recent Advances, volume 1 of Springer Tracts in Advanced Robotics, 85-108. Springer Berlin / Heidelberg.

De Luca, A. and Mattone, R. (2004). An adapt-and-detect actuator fdi scheme for robot manipulators. In Robotics and Automation, 2004. Proceedings. ICRA '04. 2004 IEEE International Conference on, volume 5, 4975 - 4980 Vol.5.

Eski, I., Erkaya, S., Savas, S., and Yildirim, S. (2010). Fault detection on robot manipulators using artificial neural networks. Robotics and Computer-Integrated Manufacturing.

Freyermuth, B. (1991). An approach to model based fault diagnosis of industrial robots. In 1991 IEEE International Conference on Robotics and Automation, volume 2, 1350-1356.

Isermann, R. (2005). Model-based fault-detection and diagnosis status and applications. Annual Reviews in Control, 29(1), $71-$ 85.

Kato, K. (2000). Wear in relation to friction - a review. Wear, 241(2), $151-157$.

McIntyre, M., Dixon, W., Dawson, D., and Walker, I. (2005). Fault identification for robot manipulators. Robotics, IEEE Transactions on, 21(5), 1028-1034.

Roylance, B. (2005). Ferrography-then and now. Tribology International, 38(10), $857-862$.

Seifert, W. and Westcott, V. (1972). A method for the study of wear particles in lubricating oil. Wear, 21(1), $27-42$.

Van Trees, H.L. (2001). Detection, Estimation and Modulation Theory, Part I. Wiley.

Vemuri, A.T. and Polycarpou, M.M. (2004). A methodology for fault diagnosis in robotic systems using neural networks. Robotica, $22(04), 419-438$ 